\title{
Understanding Soybean Cyst Nematode HG Types and Races
}

Gregory L. Tylka, Department of Plant Pathology and Microbiology, Iowa State University, Ames 50011

Accepted for publication 25 May 2016. Published 20 June 2016.

Tylka, G. L. 2016. Understanding soybean cyst nematode HG types and races. Plant Health Prog. 17:149-151.

It has been more than 45 years since the race test was created to describe and differentiate the ability of populations of the soybean cyst nematode (SCN), Heterodera glycines, to reproduce on resistant soybean cultivars (Golden et al. 1970). Nearly 15 years have passed since the updated adaptation of the race test, the HG type test, was established (Niblack et al. 2002). Unfortunately, confusion still exists about the concept of $\mathrm{SCN}$ race and $\mathrm{HG}$ type. Questions from researchers and from agribusiness and extension professionals who advise farmers include:

- When will there be a molecular test for HG type (or SCN race)?

- How many HG types (or races) are there in a field?

- Do seed treatments work against all HG types (or races)?

These seemingly logical questions of practical significance are unanswerable if one understands the concept of HG type and $\mathrm{SCN}$ race. Following is a comparison drawn between the current SCN HG type test and a hypothetical test for human food sensitivities that may provide a new perspective and make HG types more easily understood and used.

\section{A HYPOTHETICAL HUMAN EQUIVALENT TO THE HG TYPE TEST}

Some human beings cannot digest the sugar lactose in dairy products, some are allergic to a specific protein in peanuts, some cannot digest proteins called gluten in wheat, and some are allergic to shellfish (Venter 2009). An individual human being may have any one of these food sensitivities or a combination of them. The frequency of occurrence of food sensitivities in populations of human beings can vary among geographical regions and ethnic groups (Venter 2009).

Suppose that for business purposes there was a need to know how common these four food sensitivities were in order to more efficiently plan for food sales at upcoming cultural or sporting events being held at a local theater or stadium. It would be impossible to test every single person for food sensitivities in a population of people who might attend such an event. However, people attending an actual event could be randomly selected and tested to determine if they were sensitive to dairy, peanuts, wheat, and/or shellfish as a way to estimate how common the food

Corresponding author: Gregory L. Tylka. Email: gltylka@iastate.edu.

\section{doi:10.1094/PHP-PS-16-0615}

(C) 2016 The American Phytopathological Society

Plant Health Perspectives are published at the discretion of the Editor in Chief, and are evaluated for importance, relevance, and appropriateness. The views expressed are those of the author. sensitivities were in people who might attend such an event in the future. This type of food sensitivity testing could be called the "HS type test" with "HS" representing Homo sapiens (just as the "HG" in HG type test represents Heterodera glycines). The results of the testing conducted on the people might be:

- $21 \%$ had an adverse reaction to eating dairy products;

- $7 \%$ had an adverse reaction to eating food containing peanuts;

- $19 \%$ had an adverse reaction to eating foods containing wheat; and

- $2 \%$ had an adverse reaction to eating shellfish.

If random samples of the people attending the event were selected and if there was no bias or unusual pattern of how people were seated at the event, the results of the "HS type test" would provide an indication to food vendors of the food sensitivities of all of the people at that event that day. The results would indicate how common those food sensitivities might be in human beings attending such an event in the future.

Conducting an HG type test on an SCN population in a soil sample collected from a field is very similar to the hypothetical "HS type test" described above. The percentages of an SCN population that can reproduce on seven $\mathrm{HG}$ type indicator soybean lines, which are used to develop SCN-resistant soybean breeding lines and cultivars (Niblack et al. 2002), are determined in an HG type test. The results are based on the capabilities of individual nematodes in a sample being representative of the much larger nematode population that is present in the field.

Both the hypothetical, human "HS type test" and the real SCN HG type test determine the frequency of phenotypes of individuals in a population. A phenotype is an observable or measurable characteristic or ability of an organism-such as having an adverse reaction to eating foods containing dairy products, peanuts, wheat, or shellfish, or the ability of an individual SCN to develop and reproduce on the seven HG type indicator soybean lines.

\section{USING INDEX NUMBERS IN HG TYPE DESIGNATIONS PROVIDES USEFUL INFORMATION}

In addition to determining percent $\mathrm{SCN}$ reproduction on the seven HG type indicator soybean lines, the HG type test includes the soybean indicator line index numbers (Table 1) in the type designation, such as $\mathrm{HG}$ type 2.7, for all indicator lines on which the SCN population had $10 \%$ or more reproduction relative to a susceptible soybean cultivar. Golden et al. (1970) decided that an SCN population with $10 \%$ or more reproduction would be considered "positive" for that resistance line because it was a sufficient amount of reproduction to allow the SCN population to "build up quickly" on resistant soybean lines (Riggs 1988). The $10 \%$ reproduction value (called a "female index") was retained as a critical level in the current HG type test (Niblack et al. 2002). A 
female index of no more than $10 \%$ also is considered the amount of SCN reproduction allowed by a resistant soybean line or cultivar (Schmitt and Shannon 1992).

Index numbers could be assigned to the foods used in the hypothetical "HS type test." Dairy could be designated as indicator food \#1, peanuts could be \#2, wheat could be \#3, and shellfish could be \#4 (Table 1). The population of people tested at the event could be described based on the food sensitivities that were present in at least $10 \%$ of the population (if $10 \%$ was deemed to be a critical level for the food vendors). In the example given above, the population would be an "HS type 1.3" because more than $10 \%$ of the tested samples of human beings were sensitive to indicator foods \#1 (dairy) and \#3 (wheat).

Those working to manage SCN primarily need to remember that HG type test indicator line \#2 corresponds to PI 88788 (Table 1) because PI 88788 is the source of resistance available in more than $95 \%$ of currently available SCN-resistant soybean cultivars (Tylka and Mullaney 2015). So, an SCN population with an HG type with the number 2 in it (such as 2 or 2.7 or 1.2.5.7) has increased reproduction ( $>10 \%$ female index) on PI 88788, and the performance of an SCN-resistant soybean cultivar with the PI 88788 source of resistance may be less than optimum. The other numbers in the HG type designation represent other HG type indicator lines (Table 1) on which reproduction was $10 \%$ or greater. If an SCN population does not have a female index greater than $10 \%$ on any of the seven HG type indicator lines, it is designated as HG type 0 (Niblack et al. 2002).

The results of the hypothetical "HS type test" would have useful, practical significance, just like the HG type test. Knowing the "HS type" of the people at the event would allow food vendors to anticipate what types of food items they likely should sell in the future. For example, a food vendor likely would not sell as much pizza to an audience that is "HS type 1.3" as to an audience with no food sensitivities (designated "HS type 0") because at least $10 \%$ of the "HS type 1.3" audience could not eat (i.e., was sensitive to) cheese and wheat used in pizza.

\section{TYPE TESTS DESCRIBE POPULATIONS, NOT INDIVIDUALS}

In the hypothetical "HS type test" described above, no individual human being is designated an "HS type 1.3" because "1.3" means that $10 \%$ or more of the sampled population was not able to eat dairy (indicator food \#1) and wheat (indicator food \#3). An individual person attending the event in the example may be able to eat dairy and wheat even though the overall population was found to be "HS type 1.3." And in fact, most people attending the event in the example would be able to eat dairy and wheat because the percentage of individuals that were found to be intolerant to dairy and wheat in the testing was no more than $21 \%$.

Similarly, no single SCN individual can be a specific HG type or race (such as HG type 2 or race 1). An SCN individual in a population could be unable to reproduce on PI 88788 even though the HG type of the SCN population was type 2 . An HG type 2 designation does not mean that $100 \%$ of the SCN population can reproduce on PI 88788, only that at least $10 \%$ can (more specifically, at least $10 \%$ of the number that can reproduce on a susceptible soybean cultivar). The HG type (or $\mathrm{SCN}$ race) designation does not describe any SCN individual, only the SCN population.

\section{ACTUAL PERCENTAGES TELL THE WHOLE STORY}

The food vendors for the event in the hypothetical "HS type testing" scenario described above would need to consider the

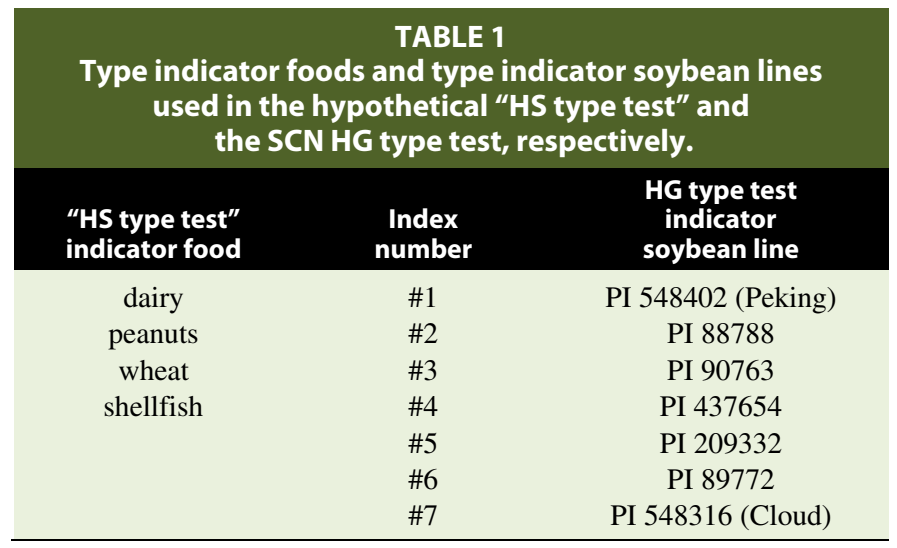

actual frequency percentages of sensitivity to the four food index items to fully understand how many event attendees will not be able to buy and eat pizza (containing a wheat crust and lots of cheese). Likewise, soybean researchers, farmers, and agribusiness and extension personnel who advise farmers must consider the actual female indices in an SCN HG type test to fully understand the parasitic capabilities or virulence phenotype of an SCN population in a field in which a resistant soybean cultivar will be grown. The female index on a type indicator soybean line in an HG type test can be thought of as somewhat of an "aggressiveness index" for the SCN population. That is, an HG type 2 SCN population with a female index of 52 on PI 88788 would be more aggressive (i.e., would reproduce more) on an SCN-resistant soybean cultivar with PI 88788 resistance than a type $2 \mathrm{SCN}$ population with a female index of 15 .

\section{TYPE IS NOT RELATED TO ANY OTHER CHARACTERISTIC}

Human beings with the same food sensitivities (i.e., the same "HS type") don't have the same eye color or height or shoe size or blood type. In other words, eye color, height, shoe size, and blood type are unrelated to food sensitivities in humans. Similarly, the HG type of an SCN population is not known to correlate with the frequency of the nematode population's preference for a soil texture or type, the size of eggs or juveniles, vulnerability to a nematicide, or any other non-parasitic characteristic of the nematode population. The HG type of an SCN population is defined only by its ability to reproduce on the seven HG type indicator soybean lines. The indicator lines are the sources of SCN resistance used in soybean germplasm lines or cultivars that have been registered (Niblack et al. 2002).

\section{SUMMARY}

The hypothetical "HS type test" described above could be used to describe a sample of human beings attending a large cultural or sporting event, and the real-life HG type test describes populations of SCN in soil samples collected from infested fields. Type and race descriptions do not apply to individuals; they describe populations of individuals. And it is knowledge of the characteristics (phenotypes) of populations of humans and SCN that is useful. As explained herein, the hypothetical "HS type test" could give food vendors useful information for planning food sales at large cultural or sporting events. Similarly, the HG type test gives useful information to farmers and agribusiness and extension professionals who advise them for developing, implementing, and monitoring the success of an SCN management plan using SCN-resistant soybean cultivars. 


\section{LITERATURE CITED}

Golden, A. M., Epps, J. M., Riggs, R. D., Duclos, L. A., Fox, J. A., and Bernard, R. L. 1970. Terminology and identity of infraspecific forms of the soybean cyst nematode (Heterodera glycines). Plant Dis. Rep. 54:544546.

Niblack, T. L., Arelli, P. R., Noel, G. R., Opperman, C. H. Orf, J. H., Schmitt, D. P., Shannon, J. G., and Tylka, G. L. 2002. A new classification scheme for genetically diverse populations of Heterodera glycines. J. Nematol. 34:279-288.
Riggs, R. D. 1988. Races of Heterodera glycines. Nematropica 18:163-170. Schmitt, D. P., and Shannon, G. 1992. Differentiating soybean responses to Heterodera glycines races. Crop Sci. 32:275-277.

Tylka, G. L., and Mullaney, M. P. 2015. Soybean cyst nematode-resistant soybeans for Iowa. Coop. Ext. Publ. PM 1649. Iowa State Univ., Ames.

Venter, C. 2009. Classification and prevalence of food hypersensitivity. Pages 3-21 in: Food Hypersensitivity: Diagnosing and Managing Food Allergies and Intolerance. I. Skypala and C. Venter, eds. Wiley-Blackwell Publishing, Hoboken, NJ. 